\title{
Development trend on tunable microwave micro- antennas based on amorphous magnetic micro wires and application prospect on wireless sensing technology
}

\begin{abstract}
The microwave detection has several advantages compared with other detection methods. Microwave sensors use Doppler radar to detect moving objects using microwaves. This differs from the method used by a regular infrared sensor as the microwave is sensitive to a variety of objects that can reflect microwaves, and its sensor readings are not affected by the ambient temperature. The unique magnetic properties of amorphous magnetic microwires (AMMW), in particular its interaction with microwaves by means of Giant Magnetoimpedance effect and its magnetostrictive character allow the development of tunable $\mathrm{GHz}$ micro-antennas useful as contactless sensing elements with applicability in many fields including biomedical, civil engineering, automotive intelligent cruise-control, etc.
\end{abstract}

Keywords: remote sensor, wireless sensors, amorphous magnetic micro wires, giant magneto impedance, micro-antennas, microwaves, biosensors, stress sensors, density sensors
Volume 4 Issue 2 - 2018

\author{
Marín P,A Moya P Gueye,Archilla D \\ Institute of Applied Magnetism, Department of Materials Physics, \\ Complutense University of Madrid, Spain
}

\begin{abstract}
Correspondence: Marín P, Institute of Applied Magnetism, Department of Materials Physics, Complutense University of Madrid, Spain, Tel +34913007I83, Email mpmarin@fis.ucm.es
\end{abstract}

Received: February 15, 2018 | Published: April 04, 2018
Abbreviations: AMMW, amorphous magnetic micro wires; ICC, intelligent cruise-control, MMW, millimeter-wave; FMCW, frequency-modulation coplanar-wave, GMI, giant magneto impedance

\section{Microwave sensing technologies}

The microwave detection method has several advantages compared with other detection methods. It is able to detect objects without physical contacts, readings are not affected by temperature, humidity, noise, air, dust or light making them suitable for harsh environments and they present strong resistance to radio frequency interference. Microwaves have a wide detection range and velocity equal to the speed of light. This type of sensor is widely used in industrial, transportation and civil applications such as measuring a vehicle's speed, measuring liquid levels, automatic door motion detection, automatic washing, production line material detection, car reversing sensors, etc.The development of new techniques for the manufacture of antennas and the advance of research in the field of intelligent materials has allowed to increase the possibilities of application of microwave technologies. In the last years several developments are performed this direction. This is the case of Soli, ${ }^{1}$ a new, robust, high-resolution, low-power, miniature gesture sensing technology for human-computer interaction based on millimeterwave radar. Soli can be used for robust gesture recognition and can track gestures with sub-millimeter accuracy, running at over 10,000 frames per second on embedded hardware. Jiao et al. ${ }^{2}$ proposes a novel millimeter microwave radar sensor to detect speech signals. The utilization of a high operating frequency and a superheterodyne receiver contributes to the high sensitivity of the radar sensor for small sound vibrations. Biomedical applications are another very important field of application for this type of sensors. Noncontact accurate measurement of cardiopulmonary activity developed with a compact quadrature doppler radar sensor enables reconstruction of chest-wall movement caused by cardiopulmonary activities ${ }^{3}$ or an X-band microwave life-detection system has been developed for detecting the heartbeat and breathing of human subjects lying on the ground at a distance of about $30 \mathrm{~m}$ or located behind a cinder block wall. ${ }^{4}$ Microwave noninvasive radio frequency biosensors based on microwave impedance spectroscopy are developed to support the monitoring of biosignals and the diagnosis of chronic diseases like diabetes. ${ }^{5,6}$ In this particular case, optimized patch antennas operating at $\mathrm{GHz}$ frequency allow the detection of glucose concentration by means of glucose dependent change on blood dielectric properties.

Civil engineering is other field of application of this type of sensors. In particular ground-based radar interferometry is an increasingly popular technique for monitoring civil infrastructures. ${ }^{7}$ Many research groups, professionals, and companies have tested it in different operative scenarios. Radar interferometry is a powerful remote sensing technique, able to detect small displacement at great distance. Groundbased radar interferometry is a measurement technique recently proposed for static testing and dynamic testing of infrastructures. It has the unique advantage to provide global information on the structure under test, with the drawback that it is often not easy to localize with precision in the structure the measured displacement as all the points in the same resolution cell can contribute to it. The technique has been demonstrated effective in bridges, culverts, dams, towers, chimneys, pylons, antenna masts, lighting towers, streetlight, and even multistorey buildings. For all these application the radar is a valuable tool for contributing to assess the health of the structure; nevertheless it should be clearly stated that the measurements of displacements and natural frequencies, as well as the modal shapes, never give directly structural information. The health evaluation of a civil structure always depends essentially on the reliability of the specific (static or dynamic) model. The instrumental data are only 
possible inputs, as well as the survey and the materials tests. Other interesting systems are automotive intelligent cruise-control (ICC). ${ }^{8}$ If they have to be successful in the marketplace, they must provide robust performance in a complex roadway environment. Inconveniences caused by reduced performance during inclement weather, interrupted performance due to dropped tracks, and annoying nuisance alarms will not be tolerated by the consumer, and would likely result in the rejection of this technology in the marketplace. To solve this problem ME Russell et al. ${ }^{8}$ propose an all-weather automotive millimeter-wave (MMW) radar sensor that uses a frequency-modulation coplanar-wave (FMCW) radar design capable of acquiring and tracking all obstacles in its field of view. One way to expand the possibilities of microwaves based detection is its combination with smart sensors embedded in the target. This combination has the potential to fundamentally change the way civil infrastructure, biomedical, etc. systems are monitored, controlled, and maintained. The design, fabrication, and construction of smart structures are one of the ultimate challenges to engineering researchers today.

\section{Applications prospect of magnetic micro wires on wireless sensing technology}

The proposed smart element to be combined with microwave technology is an Amorphous Magnetic Microwire (AMMW). Previous studies have demonstrated how a GHz electromagnetic wave can be disturbed by AMMW.

This material is one of the most widely studied soft magnetic materials. AMMW are fabricated by means of extracting melt-spinning Taylor technique. ${ }^{9}$ These AMMW are composed by a metallic core and a Pyrex cover both in the micrometre range. The metallic core provides the magnetic behaviour while the cover has a protective and stress-inducting function. Many properties of these materials have been deeply studied both from the point of view of the basic physics and the applications..$^{10}$ This is the case of the giant magnetoimpedance effect (GMI). ${ }^{11}$ In the frequency range of $\mathrm{GHz}$, some experimental and theoretical studies of the effect of the magnetization on the scattering properties of a single microwire, have been recently developed. ${ }^{12,13}$ The microwave scattering by a single microwire depends on the magnetic permeability with sufficient strength to be experimentally detected as an effect of the GMI. Furthermore, this dependence was also used to show the potential of such microwire as a wireless field and/or stress sensor. These experimental results are followed by a theoretical approach where the influence of the microwire magnetic state in its microwave reflection features is taken into account. Based in these studies, further experimental work shows an application of such microwire as a wireless stress sensor with the particular application of pressure detection in a hydraulic circuit simulating cardiovascular conditions. ${ }^{14,15}$ Some recent studies reveal the possibility of using such a kind of AMMW to measure density and mechanical stress without contacts. Microwave scattered intensity produced by a single microwire can be controlled by tuning its permeability. This permeability may be modified with the application of an external bias field, leading to non-negligible effects in the scattering. In addition, if the cylinder is magnetostrictive, the scattering is also sensitive to mechanical stresses. These experimental results are promising for future developments in this field including in situ and in vivo biomedical magnetoelastic experiments, taking advantage of the biocompatible nature of the microwire Pyrex cover. So far, these kinds of measurements were only possible by using GMI or Magnetoelastic Resonance based devices. However, the use of GHz frequency devices would allow the development of sensors operating at much longer distances and with a higher information transfer rate.

\section{Conclusion}

New developments on microwaves based sensing technologies should open the possibilities and capacities of robots for multiple and complex applications. For instance, it turns out that the microwave behavior of finite ferromagnetic Co-based microwires could be useful for the construction of tags. The information will be codified in the length of the AMMW. The lecture principle of the tag will be based on the tuning of the induced electric current along the microwire, and thus of the scattering properties. Besides, if a magnetic field is locally applied, the code encrypted by the microwire tag will change depending on the saturated microwires in the tag. Therefore, ferromagnetic microwires have a large potential for novel devices as wireless sensing element, working on microwaves, of magnetic field as well as the codification of information in the form of a tag. ${ }^{13}$ In particular, in vitro experiments performed with magnetoelastic microwire have been able to locate and quantify the stenosis caused in bovine artery and in a lateroterminal anastomosis of PTFE with a high statistical power. ${ }^{15}$

\section{Acknowledgements}

My research project was partially or fully sponsored by (Ministerio de Economía y Competitividad y Comunidad de Madrid) with grant number (MAT2015-67557-C2-1-P, S2013/MIT-2850 NANOFRONTMAG-CM).

\section{Conflict of interest}

The authors declare there is no conflict of interest.

\section{References}

1. Jaime Lien, Nicholas Gillian, M Emre Karagozler, et al. Soli: ubiquitous gesture sensing with millimeter wave radar. ACM Trans Graph. 2016;35(4):1-19.

2. Mingke Jiao, Guohua Lu, Xijing Jing, et al. A novel radar sensor for the non-contact detection of speech signals. Sensors (Basel). 2010;10(5):4622-4633.

3. Wei Hu, Zhangyan Zhao, Yunfeng Wang, et al. Noncontact accurate measurement of cardiopulmonary activity using a compact quadrature doppler radar sensor. IEEE Trans Biomed Eng. 2014;61(3):725-735.

4. Chen KM, Misra D, Wang H, et al. An X-band microwave life-detection system. IEEE Trans Biomed Eng. 1986;33(7):697-702.

5. Yilmaz T, Foster R, Hao Y. Towards accurate dielectric property retrieval of biological tissues for blood glucose monitoring. IEEE Transactions on Microwave Theory and Techniques. 2014;62(12): 3193-3204.

6. Yilmaz T, Foster R, Hao Y. Broadband tissue mimicking phantoms and a patch resonator for evaluating noninvasive monitoring of blood glucose levels. IEEE Trans on Ant and Prop. 2014;62(6):3064-3075.

7. Pieraccini M. Monitoring of civil infrastructures by interferometric radar: a review. Scientific World Journal. 2013;1-8.

8. Russell ME, Crain A, Curran A, et al. Millimeter-wave radar sensor for automotive intelligent cruise control (ICC). IEEE Transactions on Microwave Theory and Techniques. 1997;45(12):2444-2453.

9. Taylor GF. A method of drawing metallic filaments and a discussion of their properties and uses. Physical Review. 1924;23:655-658. 
10. Marín P, Vázquez $\mathrm{M}$, Arcas $\mathrm{J}$, et al. Thermal dependence of magnetic properties in nanocrystalline $\mathrm{FeSiBCuNb}$ wires and microwires. J of Magn Magn Mat. 1999;203(1-3):6-11.

11. Thiabgoh O, Shen H, Eggers T, et al. Enhanced high-frequency magnetoimpedance response of melt-extracted $\mathrm{Co}_{6925} \mathrm{Fe}_{425} \mathrm{Si}_{13} \mathrm{~B}_{135}$ microwires subject to Joule annealing. Journal of Science: Advanced Materials and Devices. 2016;1(1):69-74.

12. Herrero-Gómez C, Aragón AM, Hernando-rydings M, et al. Stress and field contactless sensor based on the scattering of electromagnetic waves by a single ferromagnetic microwire. Applied Physics Letters. 2014;105(9):1-4.
13. Hernando A, López-Domínguez V, Ricciardi E, et al. Tuned scattering of electromagnetic waves by a finite length ferromagnetic micro wire. IEEE Trans Ant and Prop. 2016;64(3):1112-1115.

14. Aragón A, Hernando-Rydings M, Hernando A, et al. Liquid pressure wireless sensor based on magneto strictive micro wires for applications in cardiovascular localized diagnostic. AIP Advances. 2015;5(8):1-7.

15. Hernando Rydings M, Marín Palacios P, Aragón-Sánchez AM, et al. Development of a telemetric system for postoperative follow-up of vascular surgery procedures: in vitro model. J Am Heart Assoc. 2016;5(7):e003608. 